\title{
Three-phase intersection points in monolayers
}

\author{
John P. Hagen, Harden M. McConnell
}

\begin{abstract}
Some phospholipid/dihydrocholesterol Langmuir monolayers form coexisting liquid phases. Gas domains form at the interface between the phospholipid-rich and dihydrocholesterol-rich liquid phases when these monolayers undergo expansion to low surface pressure. Analysis of the domain shapes thus formed yields the relative line tensions of the gas/phospholipid, gas/dihydrocholesterol, and phospholipid/dihydrocholesterol phase interfaces.
\end{abstract}

\section{Introduction}

Lipid monolayers have been extensively observed using fluorescence microscopy [1-3]. Certain properties of these monolayers have been accounted for by modeling them as two-dimensional arrays of dipoles [4]. Lipid domain shapes of the monolayers are explained by electrostatic repulsion among the dipoles and line tension along the interface between phases, when two phases are present. Calculations based on this theory require experimental measurement of dipole density and line tension. Measurements have been made of the line tension between the liquid and the solid phases of phospholipid [5], fluorescently-labeled stearic acid [6], and protonated amine [7] monolayers. The gas/liquid line tension of ethyl heptadecanoate has also been reported [8], as well as the liquid/ liquid line tension in monolayers composed of cholesterol/phospholipid mixtures [9]. However, there has been no measurement of the relative values of line tensions in a three-phase system. The present paper describes such measurements.
Many studies have examined the behavior of monolayers composed of mixtures of phospholipids and cholesterol or cholesterol analogs [10-12]. These monolayers are especially valuable in modeling biological membranes; eukaryotic cell membranes are composed of similar mixtures of cholesterol and phospholipids. Some of these mixtures, at certain temperatures and surface pressures, separate into phospholipid-rich and cholesterolrich liquid phases [10]. A third, gaseous phase forms at the interface between the phospholipidrich and cholesterol-rich phases when these monolayers undergo expansion to low surface pressure. A fluorescently-labeled phospholipid probe partitions unevenly into the three phases, allowing them to be distinguished in a fluorescence microscope. There are three interfaces between the various phases, each with a different line tension. Since the points where the three interfaces come together are static, the relative line tensions of the three interfaces may be calculated by force balance. 


\section{Materials and methods}

The phospholipid diphytanoyl-L- $\alpha$-phosphatidylethanolamine (DPhPE) and the fluorescent lipid probe $N$-(7-nitro-2-1,3-benzoxadiazol-4-yl)dipalmitoyl-L- $\alpha$-phosphatidylethanolamine (NBDDPPE) were purchased from Avanti Polar Lipids, Inc. The synthetic cholesterol analog dihydrocholesterol (DChol) was purchased from Sigma. All chemicals were used without further purification.

All experiments were done at $20-22^{\circ} \mathrm{C}$. The subphase was distilled, deionized water. Compression and expansion were carried out with a movable barrier and surface pressure was measured with a Wilhelmy plate. The monolayer was viewed with a Zeiss epifluorescence microscope fitted with a Cohu low-level video camera. The monolayer images were recorded on a JVC BR601MU video recorder.

The lipids were spread from $1 \mathrm{mM}$ chloroform solution containing $1 \mathrm{~mol} \%$ of the probe NBDDPPE. After spreading, the monolayer was compressed until the surface pressure reached $15 \mathrm{mN} \mathrm{m}^{-1}$. The monolayer was then expanded to $1 \mathrm{mN} \mathrm{m}^{-1}$ over a period of $30 \mathrm{~min}$ and held there for $10 \mathrm{~min}$. On further expansion to approximately $0 \mathrm{mN} \mathrm{m}^{-1}$, gas-phase domains formed. The data were collected for $10 \mathrm{~min}$ after gas domain formation. The monolayer was observed for an additional 50 min with no change in domain shape and no evident change in the configuration of the intersection points of the phase boundaries. In addition, the monolayer could be compressed and re-expanded with no hysteresis in gas domain shape. The phospholipid-rich liquid phase appeared white, the DChol-rich liquid phase appeared gray, and the gas phase appeared black, in accordance with the amount of fluorescent probe that partitioned into the various phases. Three monolayers, each of different lipid composition, were recorded. As image processing can result in a loss in resolution, measurements were made directly from the monitor screen as the previously recorded images were replayed. 10 measurements of the values of $r_{1}, r_{2}, r_{3}$, and $d$ (see below) were made for the monolayers with 70 and $90 \mathrm{~mol} \%$ DChol. As the monolayer with $60 \mathrm{~mol} \%$ DChol displayed two different morphologies (see below), 10 measurements of each form were made.

\section{Results and discussion}

The monolayer consists of domains of DCholrich phase surrounded by a background of phospholipid-rich phase when the mixture has less DChol than the critical point composition (approximately $60 \%$ DChol). This will be referred to as case I. At DChol concentrations higher than the critical composition, the monolayer consists of domains of phospholipid-rich phase surrounded by a background of DChol-rich phase. This will be referred to as case II.

The gas phase nucleates at the boundaries of the liquid-phase domains when the monolayer is greatly expanded (Fig. 1). In case I, the gas domains expand outward from the edges of DCholrich domains into the phospholipid-rich phase (Fig. 2). Often, several gas domains nucleate at the edge of the same DChol-rich domain; the shapes thus formed are reminiscent of pentadecanoic acid monolayers at the triple point [13]. In case II, the gas-phase domains expand inwards from the edges of the phospholipid-rich domains (Fig. 3). In mix-

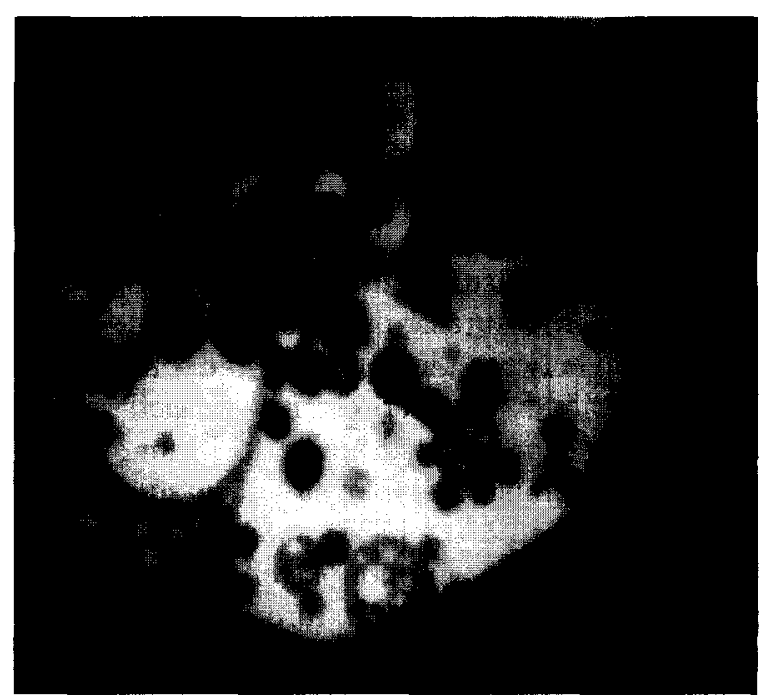

Fig. 1. Fluorescence micrograph of monolayer showing coexisting DChol-rich (gray), phospholipid-rich (white), and gas (black) phases. 

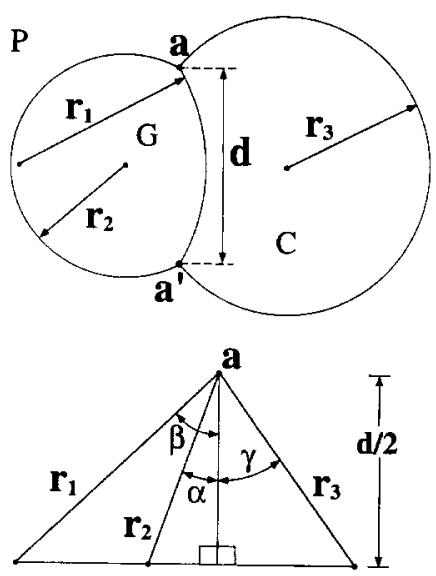

Fig. 2. Case I morphology. $\mathrm{P}$ is the phospholipid-rich phase, $\mathrm{C}$ is the DChol-rich phase, $\mathrm{G}$ is the gas phase, $r_{2}$ is the radius of the gas/phospholipid-rich phase boundary, $r_{1}$ is the radius of the gas/DChol-rich phase boundary, $r_{3}$ is the radius of the DChol/phospholipid-rich phase boundary, points a and a' are the three-phase intersections, and $d$ is the distance between points and and $a^{\prime}$. The geometric construction below the gas domain is drawn to clarify the trigonometric relationships.
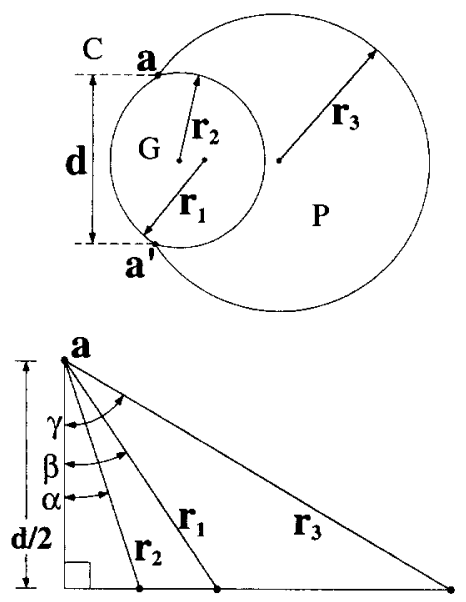

Fig. 3. Case II morphology. $\mathbf{P}$ is the phospholipid-rich phase, $\mathrm{C}$ is the DChol-rich phase, $\mathrm{G}$ is the gas phase, $r_{2}$ is the radius of the gas/phospholipid-rich phase boundary, $r_{1}$ is the radius of the gas/DChol-rich phase boundary, $r_{3}$ is the radius of the DChol/phospholipid-rich phase boundary, points a and $a^{\prime}$ are the three-phase intersections, and $d$ is the distance beween points $a$ and $a^{\prime}$. The geometric construction below the gas domain is drawn to clarify the trigonometric relationships.

tures near the critical composition, both morphologies were present (Fig. 1). In both cases the shapes correspond to three circular arcs of unequal radii.
When the gas phase forms in the presence of the two liquid phases, two arrangements are possible. The gas phase may spread to wet the interface between the two liquid phases or the three phases may meet at a point. The gas phase would wet the interface between the two liquid phases if Antonow's rule [14] were obeyed:

$\lambda_{3}=\lambda_{1}+\lambda_{2}$

where $\lambda_{3}$ is the line tension between the two liquid phases, and $\lambda_{2}$ and $\lambda_{1}$ are the two liquid/gas line tensions. There is no wetting (the three phases will meet at a point) if the phases obey the following inequality:

$\lambda_{1}<\lambda_{2}+\hat{\lambda}_{3}$

where $\lambda_{1}$ is the largest line tension, and $\lambda_{2}$ and $\lambda_{3}$ are two smaller line tensions.

In our experiments wetting did not occur. Therefore, each of the line tensions is smaller than the sum of the other two. In order to analyze our data the assumption was made that the arcs are circular with no additional bending on a microscopic scale. This assumption is discussed further in Appendix B.

The three different interfaces between the various phases have unequal line tensions. These interfaces meet at points a and a' (Figs. 2 and 3). As these points are static (within the resolution of the microscope, $<1 \mu \mathrm{m}$ ), the vector sum of the line tension forces must be zero. The angles at which the phase boundaries meet were calculated from the radii of the three arcs and from the distance $d$ between points $a$ and $a^{\prime}$ :

$d / 2 r_{2}=\cos \alpha$

$d / 2 r_{1}=\cos \beta$

$d / 2 r_{3}=\cos \gamma$

where $\alpha, \beta$, and $\gamma$ are the angles between a line going through points a and $\mathrm{a}^{\prime}$, and $r_{2}, r_{1}$, and $r_{3}$, respectively, as shown in Fig. 2. These angles allow calculation of the relative line tensions by force balance in two dimensions:

Case I

$$
\begin{aligned}
& \lambda_{1} \sin \beta=\lambda_{2} \sin \alpha+\lambda_{3} \sin \gamma \\
& \lambda_{2} \cos \alpha=\lambda_{1} \cos \beta+\lambda_{3}+\cos \gamma
\end{aligned}
$$




\section{Case II}

$\lambda_{1} \sin \beta=\lambda_{2} \sin \alpha+\lambda_{3} \sin \gamma$

$\lambda_{1} \cos \beta=\lambda_{2} \cos \alpha+\lambda_{3}+\cos \gamma$

where $\lambda_{1}, \lambda_{2}$, and $\lambda_{3}$ are the line tensions between the DChol/gas, phospholipid/gas, and DChol/ phospholipid phase interfaces, respectively. The morphologies for Case I and Case II are given in Figs. 2 and 3.

According to Gibbs' phase rule, the compositions of the three coexisting phases are fixed. A schematic phase diagram is shown in Fig. 4. The line tension is a function of the composition of the adjacent phases. Since the compositions of the phases are the same regardless of the net monolayer composition, there should be no dependence of line tension on this composition. This was found to be the case (Figs. 5 and 6 ). The average value for $\lambda_{1} / \lambda_{3}$ was $2.5 \pm 0.3$ and for $\lambda_{2} / \lambda_{3}$ was $1.7 \pm 0.3$.

Note that the contact angles of the various phases vary as $\gamma-\beta, \beta-\alpha$, and $\gamma-\alpha$. The angles $\alpha, \beta$, and $\gamma$ themselves depend on the ratio of the areas of the two phases comprising the domain. Fig. 7 illustrates this point where, from top to bottom, there is an increasing area of gas. In constructing this figure, it is convenient to use the following equation that relates the area ratio $R$ of gas to phospholipid-rich phase, to the angles $\alpha, \beta$, and $\gamma$ :

$R=\frac{\frac{2 \alpha+\pi}{2 \cos ^{2} \alpha}+\frac{\pi-2 \beta}{2 \cos ^{2} \beta}-\tan \alpha+\tan \beta}{\frac{2 \gamma+\pi}{2 \cos ^{2} \gamma}-\frac{2 \alpha+\pi}{2 \cos ^{2} \alpha}-\tan \alpha+\tan \gamma}$

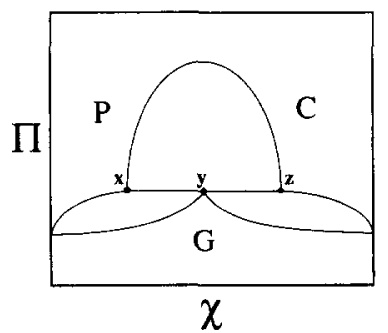

Fig. 4. Schematic of the lipid/DChol phase diagram. $P$ is the phospholipid-rich phase, $\mathrm{C}$ is the DChol-rich phase, $\mathrm{G}$ is the gas phase, $\Pi$ is the surface pressure, and $\chi$ is the composition (mole fraction DChol). $x, y$, and $z$ are the compositions of the three coexisting phases.

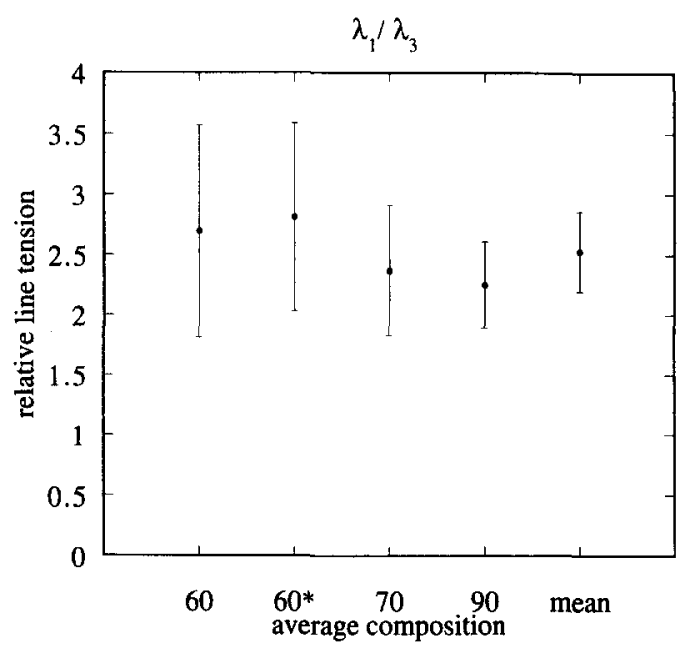

Fig. 5. Relative line tension, $\lambda_{1} / \lambda_{3}$, at different system compositions. Line tension is unaffected by system composition. The composition numbers refer to the mol\% DChol. $60^{*}$ refers to data taken from gas domains of case I morphology. All other data were taken from gas domains of case II morphology. Data are shown with $95 \%$ confidence intervals.

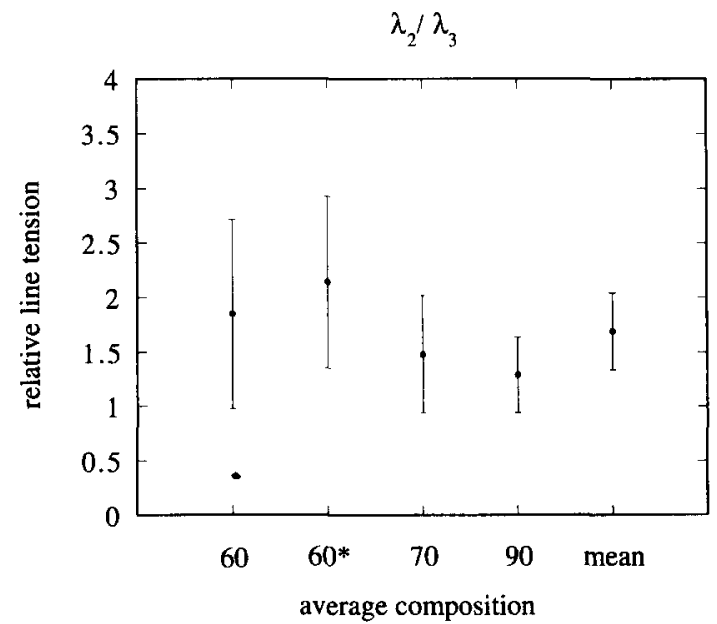

Fig. 6. Relative line tension, $\lambda_{2} / \lambda_{3}$, at different system compositions. Line tension is unaffected by system composition. The composition numbers refer to the mol\% DChol. $60^{*}$ refers to data taken from gas domains of case I morphology. All other data were taken from gas domains of case II morphology. Data are shown with $95 \%$ confidence intervals

Eq. (10) can be solved simultaneously with Eqs. (8) and (9) for various values of $R$, to obtain $\alpha, \beta$, and $\gamma$. The resulting shapes for $R$ values of 0.125 , 1 , and 8 are shown in Fig. 7. Note that the contact 


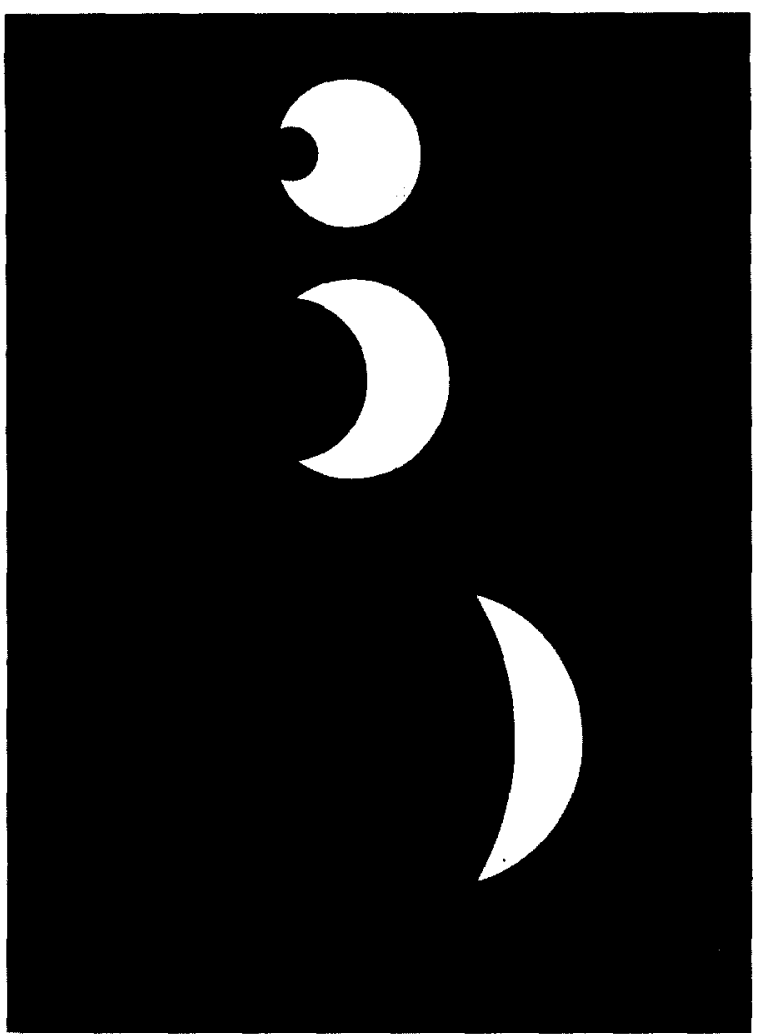

Fig. 7. The three-phase intersection.

angles remain the same as the gas domains grow. The shapes shown in Fig. 7 are similar to those observed experimentally.

The analysis given in the present paper bears on both physical (mechanical) and chemical equilibrium in monolayers. When we find that a single three-phase intersection point has a stable geometry over a $50 \mathrm{~min}$ period of time, we can be sure that the system is mechanically stable over that period of time. Since we are dealing with two liquid phases and one gas phase, this mechanical stability implies that the line tensions $\lambda_{1}, \lambda_{2}$, and $\lambda_{3}$ are constant over this same period of time. Further, since line tensions are sensitive to the composition of the phases (as well as the presence of impurities), we conclude that there is also (local) equilibrium of chemical composition over this same period of time. This view is also supported to the extent that the line tension ratios in Figs. 5 and 6 are constant, since a phase diagram such as that sketched in
Fig. 4 implies constancy of chemical composition of the three phases.

As a final point in these considerations, note that it is clear from recent work on the hydrodynamics of shape changes in fluid lipid monolayers that unbalanced pressure and electrostatic forces acting on lipid domains are quickly relieved for simple domain shapes [15]. However, there are substantial activation energies for domain fission and fusion for some lipid compositions, and this may explain the persistence of complex patterns such as those seen in Fig. 1.

\section{Acknowledgments}

We thank Dr. Ka Yee Lee for helpful discussions. This work was supported by National Science Foundation Grant No. NSF MCB 9316256.

\section{Appendix A}

It is of interest to consider the surface pressure difference between the various phases due to the curvature of the phase boundaries and line tension. This yields the following equations:

$$
\begin{aligned}
& \pi_{\mathrm{c}}=\pi_{\mathrm{g}}+\kappa_{1} \lambda_{1} \\
& \pi_{\mathrm{g}}=\pi_{\mathrm{p}}+\kappa_{2} \lambda_{2} \\
& \pi_{\mathrm{p}}=\pi_{\mathrm{c}}+\kappa_{3} \lambda_{3}
\end{aligned}
$$

where $\pi_{\mathrm{c}}, \pi_{\mathrm{g}}$, and $\pi_{\mathrm{p}}$ are the pressures in the DChol, gas, and phospholipid phases, and $\kappa$ is the curvature of the interface. The elimination of pressure from these equations yields the following:

$\kappa_{1} \lambda_{1}+\kappa_{2} \lambda_{2}+\kappa_{3} \lambda_{3}=0$

Thus, one of the curvatures must be opposite in sign from the other two. It can be seen from Fig. 2 that in Case I this is $\kappa_{2}$. Therefore

$\frac{\lambda_{2}}{r_{2}}=\frac{\lambda_{1}}{r_{1}}+\frac{\lambda_{3}}{r_{3}}$

Eq. (A4), together with the definitions Eqs. (3)-(5), leads to Eq. (7). In Case II it is $\kappa_{1}$ that has the 
opposite sign (see Fig. 3). Therefore

$\frac{\lambda_{1}}{r_{1}}=\frac{\lambda_{2}}{r_{2}}+\frac{\lambda_{3}}{r_{3}}$

This leads to Eq. (9).

\section{Appendix B}

The above discussion assumes that line tension alone determines the shape of the three-phase intersection at points a and $a^{\prime}$. However, dipole repulsions at the air/water interface can affect the shapes of the interface between two-dimensional lipid domains [1-4]. In the presence of these dipolar forces, Eqs. (A1)-(A3) remain valid for pairs of points on opposite sides of the phase boundary, but the pressures $\pi_{\mathrm{c}}, \pi_{\mathrm{p}}$, and $\pi_{\mathrm{g}}$ need not be uniform in each phase; thus, it might appear that these pressures cannot be eliminated from Eqs. (A1)-(A13) to yield Eq. (A5), as described above. As discussed below, mathematical elimination is possible close to the three-phase intersection point.

The chemical potential of each component in an isothermal monolayer is given by

$\mu_{1}=\mu_{1}^{(0)}+\bar{A}_{1}\left(\pi-m_{1} \epsilon\right)+f(\chi)$

where $\mu_{1}^{(0)}$ is the chemical potential of pure component $1, \bar{A}_{1}$ is the partial molar area of that component, $m_{1}$ is the dipole moment density of that component, $\epsilon$ is the long-range dipolar electric field, $\pi$ is the change in surface pressure caused by this electric field, and $f(\chi)$ is a function of composition $[16,17]$. Within each phase, the composition, dipole density, and local surface pressure all vary with the local electric field so as to keep the chemical potential constant $[16,17]$. Consider two points just inside the same domain (Fig. B1). As the points move towards the intersection, the local electric fields at the two points approach one another (there are no discontinuities in the electric field at or near the phase boundaries). Since the chemical potentials are constant, the pressures at the two points in Fig. B1 also approach one another. Therefore, the long-range dipole-dipole

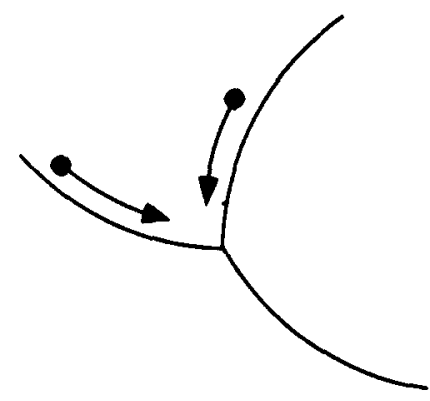

Fig. B1. Case II domains at increasing gas-phase:phospholipid-rich phase area ratios, $R$. From top to bottom, $R$ increases from 0.125 to 1 to 8 . The gas phase is shown as black, the phospholipid-rich phase is shown as white, and the cholesterolrich phase is shown as gray.

repulsion plays no role in shaping the three-phase domain geometry at the intersection point.

\section{References}

[1] C.M. Knobler, Science, 24a (1990) 870.

[2] H.M. McConnell, Annu. Rev. Phys. Chem., 42 (1991) 171.

[3] H. Möhwald, Annu. Rev. Phys. Chem., 41 (1990) 441.

[4] D.J. Keller, H.M. McConnell and V.T. Moy, J. Phys. Chem., 90 (1986) 2311.

[5] C.A. Helm, Ph.D. Thesis, Technical University of Munich, 1988, unpublished.

[6] P. Muller and F. Gallet, Phys. Rev. Lett., 67(9) (1991) 1106.

[7] K.J. Stine and D. Stratmann, Langmuir, 8(10) (1992) 2509.

[8] K.J. Stine, C.M. Knobler and R.C. Desai, Phys. Rev. Lett., 65(8) (1990) 1004.

[9] D.J. Benvegnu and H.M. McConnell, J. Phys. Chem., 96 (1992) 7101.

[10] S. Subramaniam and H.M. McConnell, J. Phys. Chem., $91(1987) 1715$.

[11] C.L. Hirshfeld and M. Seul, J. Phys. Fr., 51 (1990) 1537.

[12] D.J. Benvegnu and H.M. McConnell, J. Phys. Chem., 97 (1993) 6686.

[13] B.G. Moore, C.M. Knobler, S. Akamatsu and F. Rondelez, J. Phys. Chem., 94 (1990) 4588.

[14] J.S. Rowlinson and B. Widom, Molecular Theory of Capillarity, Clarendon Press, Oxford, 1982.

[15] H.A. Stone and H.M. McConnell, Proc. R. Soc. (London), Ser. A, 448 (1995) 97.

[16] K.Y.C. Lee, J.F. Klingler and H.M. McConnell, Science, 263 (1994) 655.

[17] K.Y.C. Lee and H.M. McConnell, Biophys. J., 8 (1995) 1740. 